PROCEEDINGS OF THE

AMERICAN MATHEMATICAL SOCIETY

Volume 128, Number 1, Pages 85-92

S 0002-9939(99)04994-1

Article electronically published on June 24, 1999

\title{
REFLECTION AND UNIQUENESS THEOREMS FOR HARMONIC FUNCTIONS
}

\author{
D. H. ARMITAGE
}

(Communicated by J. Marshall Ash)

\begin{abstract}
Suppose that $h$ is harmonic on an open half-ball $\beta$ in $R^{N}$ such that the origin 0 is the centre of the flat part $\tau$ of the boundary $\partial \beta$. If $h$ has non-negative lower limit at each point of $\tau$ and $h$ tends to 0 sufficiently rapidly on the normal to $\tau$ at 0 , then $h$ has a harmonic continuation by reflection across $\tau$. Under somewhat stronger hypotheses, the conclusion is that $h \equiv 0$. These results strengthen recent theorems of Baouendi and Rothschild. While the flat boundary set $\tau$ can be replaced by a spherical surface, it cannot in general be replaced by a smooth $(N-1)$-dimensional manifold.
\end{abstract}

\section{INTRODUCTION}

Let $x=\left(x_{1}, \ldots, x_{N}\right)$ denote a typical point of $R^{N}$, where $N \geq 2$, and let $\|\cdot\|$ be the Euclidean norm on $R^{N}$. For each positive number $r$ let

$$
\begin{aligned}
& \beta(r)=\left\{x:\|x\|<r, x_{N}>0\right\}, \\
& \tau(r)=\left\{x:\|x\|<r, x_{N}=0\right\}, \\
& \alpha(r)=\left\{\left(0, \ldots, 0, x_{N}\right) \in R^{N}: 0<x_{N}<r\right\} .
\end{aligned}
$$

A modified form of a recent theorem of Baouendi and Rothschild [1, Theorem $3]$ may be stated as follows. (The theorem was originally proved with a relatively open subset of the unit sphere in place of $\tau(r)$, but see [1, $\S 0$, final paragraph].)

Theorem BR. Let $h$ be a continuous real-valued function on $\overline{\beta(r)}$, harmonic on $\beta(r)$. If $h \geq 0$ on $\tau(r)$ and

$$
\lim _{t \rightarrow 0+} t^{-m} h(0, \ldots, 0, t)=0
$$

for each positive integer $m$, then $h=0$ on $\alpha(r) \cup \tau(\rho)$ for some $\rho \in(0, r]$.

The main result of this note is the following strengthened version of the above theorem.

Theorem 1. Let $h$ be harmonic on $\beta(r)$. If

$$
\liminf _{x \rightarrow y, x \in \beta(r)} h(x) \geq 0
$$

Received by the editors February 7, 1995 and, in revised form, March 4, 1998.

1991 Mathematics Subject Classification. Primary 31B05.

Key words and phrases. Harmonic function, reflection, uniqueness, continuation.

(C)1999 American Mathematical Society 
for each $y \in \tau(r)$ and

$$
\liminf _{t \rightarrow 0+} t^{-m}|h(0, \ldots, 0, t)|=0
$$

for each positive integer $m$, then $h=0$ on $\alpha(r)$ and

$$
\lim _{x \rightarrow y, x \in \beta(r)} h(x)=0
$$

for each $y \in \tau(r)$.

In Theorem 1 the hypotheses of Theorem BR that $h$ is continuous on $\overline{\beta(r)}$ and non-negative on $\tau(r)$ are replaced by the milder hypothesis (2). Also, (3) is a relaxation of (1).

Recall that if (4) holds for each $y \in \tau(r)$, then $h$ has a unique harmonic continuation $\bar{h}$ to the ball

$$
B(r)=\{x:\|x\|<r\}
$$

and $\bar{h}$ is obtained by reflection, that is,

$$
\bar{h}\left(x_{1}, \ldots, x_{N-1},-x_{N}\right)=-h\left(x_{1}, \ldots, x_{N}\right) \quad(x \in \beta(r)) .
$$

Thus Theorems BR and 1 may be regarded as reflection principles. Under more stringent hypotheses, these theorems become uniqueness results. For each number $d \in[0,1)$ let $C(d)$ denote the spherical cap $\left\{x:\|x\|=1, x_{N}>d\right\}$.

Corollary 1. Let $h$ be harmonic on $\beta(r)$, and suppose that (2) holds for each $y \in \tau(r)$. If there exists a sequence $\left(d_{m}\right)$ in $[0,1)$ such that

$$
\liminf _{t \rightarrow 0+} t^{-m}|h(t z)|=0 \quad\left(z \in C\left(d_{m}\right)\right)
$$

for each positive integer $m$, then $h \equiv 0$.

Corollary 2. Let $h$ be harmonic on $\beta(r)$. If

$$
\liminf _{x \rightarrow y, x \in \beta(r)} h(x) / x_{N} \geq 0
$$

for each $y \in \tau(r)$ and (3) holds for each positive integer $m$, then $h \equiv 0$.

Corollary 1 is an improvement of the half-space version of [1, Corollary 2.6], but Corollary 2 seems to have no counterpart in [1].

In $\S 5$ we give an example to show that Theorem BR (and a fortiori Theorem 1) may fail if $\beta(r)$ is replaced by a domain whose boundary is analytic near 0 and tangential at 0 to $\tau(1)$. This partially answers the conjecture [1, p. 245] that results similar to those of [1] hold for more general domains.

Baouendi and Rothschild [2] have recently generalized the main results of [1] in a direction different from that considered here.

\section{A RePRESENTATion LEMMA}

We shall need the following lemma, which may be regarded as a local version of the Poisson integral representation of a positive harmonic function on a half-space (see, e.g., Helms [4, Theorem 2.25]). 
Lemma. Suppose that $h$ is harmonic on $\beta(r)$ and satisfies (2) at each $y \in \tau(r)$. Then there exists a measure $\mu$ on $\tau(r)$ with the following property: for each $\rho \in$ $(0, r)$, there exists a harmonic function $H$ on $B(\rho)$ such that

$$
H\left(x_{1}, \ldots, x_{N}\right)=-H\left(x_{1}, \ldots, x_{N-1},-x_{N}\right) \quad(x \in B(\rho))
$$

and

$$
h(x)=x_{N} \int_{\tau(\rho)}\|x-y\|^{-N} d \mu(y)+H(x) \quad(x \in \beta(\rho)) .
$$

This lemma is known, at least tacitly, but lacking on exact reference, I indicate a proof using a technique which involves passing to a space of higher dimension. This technique is due to Huber [5] and was rediscovered and more extensively exploited by Kuran [6]. The results that we shall need are half-ball versions of [6, Lemmas 1 , 3,6 , Theorem 1], which were proved in [6] for a half-space rather than a half-ball, but which are indeed valid in the generality we require (see [6, p. 279]).

We verify first that if (2) holds for each $y \in \tau(r)$, then

$$
\liminf _{x \rightarrow y, x \in \beta(r)} x_{N}^{-1} h(x)>-\infty \quad(y \in \tau(r)) .
$$

The function $u$, defined on $\beta(r)$ by $u(x)=\min \left\{h(x), x_{N}\right\}$, is superharmonic on $\beta(r)$ and tends to 0 at each point of $\tau(r)$. It follows from [6, Lemma 3] that (10) holds with $u$ in place of $h$, and since $h \geq u$ on $\beta(r)$, (10) itself is true.

A typical point of $R^{N+2}$ is denoted by $\xi=\left(\xi_{1}, \ldots, \xi_{N+2}\right)$, and with such a point we associate the number

$$
\delta_{\xi}=\sqrt{ }\left(\xi_{N}^{2}+\xi_{N+1}^{2}+\xi_{N+2}^{2}\right) .
$$

Let $\mathcal{B}(r)$ denote the open ball of radius $r$ centred at the origin of $R^{N+2}$ and let $E=\left\{\xi: \delta_{\xi}=0\right\}$. Define $h^{*}$ on $\mathcal{B}(r) \backslash E$ by

$$
h^{*}(\xi)=\delta_{\xi}^{-1} h\left(\xi_{1}, \ldots, \xi_{N-1}, \delta_{\xi}\right) .
$$

By [6, Lemma 1], $h^{*}$ is harmonic on $\mathcal{B}(r) \backslash E$, and since (10) holds, it follows from [6, Theorem 1] that $h^{*}$ has a superharmonic extension $U$ to $\mathcal{B}(r)$. The support of the Riesz measure $\mu^{*}$ associated to $U$ is contained in $E$. Hence, by the local form of the Riesz decomposition theorem, if $0<\rho<r$, then there exists a harmonic function $H^{*}$ on $\mathcal{B}(\rho)$ such that

$$
U(\xi)=\int_{E \cap \mathcal{B}(\rho)}\|\xi-\eta\|^{-N} d \mu^{*}(\eta)+H^{*}(\xi) \quad(x \in \mathcal{B}(\rho)) .
$$

If $x \in \beta(\rho)$ and $\xi_{x}$ is defined to be the point $\left(x_{1}, \ldots, x_{N}, 0,0\right)$, then $\xi_{x} \in \mathcal{B}(\rho) \backslash E$ and $\delta_{\xi_{x}}=x_{N}$, so that

$$
\begin{aligned}
h(x) & =x_{N} h^{*}\left(\xi_{x}\right) \\
& =x_{N} U\left(\xi_{x}\right) \\
& =x_{N} \int_{E \cap \mathcal{B}(\rho)}\left\|\xi_{x}-\eta\right\|^{-N} d \mu^{*}(\eta)+x_{N} H^{*}\left(\xi_{x}\right) \\
& =x_{N} \int_{\tau(\rho)}\|x-y\|^{-N} d \mu(y)+\tilde{H}(x),
\end{aligned}
$$

where $\mu$ is the measure defined on the Borel subsets of $\tau(r)$ by

$$
\mu(F)=\mu^{*}\left(\left\{\xi \in E:\left(\xi_{1}, \ldots, \xi_{N-1}, 0\right) \in F\right\}\right)
$$


and $\tilde{H}$ is defined on $\beta(\rho)$ by $\tilde{H}(x)=x_{N} H^{*}\left(\xi_{x}\right)$. By [6, Lemma 6$], \tilde{H}$ is harmonic on $\beta(\rho)$, and since $H^{*}$ is locally bounded on $\mathcal{B}(\rho)$ it follows that $\tilde{H}$ tends to 0 at each point of $\tau(\rho)$. Therefore, by the reflection principle, $\tilde{H}$ has a harmonic continuation $H$ to $B(\rho)$ satisfying (8).

\section{Proof of Theorem 1}

Suppose that $h$ satisfies the hypotheses of Theorem 1. It is enough to fix $\rho \in(0, r)$ and to show that $h=0$ on $\alpha(\rho)$ and (4) holds for each $y \in \tau(\rho)$.

According to the lemma, $h$ has the representation (9) on $\beta(\rho)$. Since the function $H$ in (9) is harmonic on $B(\rho)$, there exists a series $\sum_{j=0}^{\infty} H_{j}$, where $H_{j}$ is a homogeneous harmonic polynomial of degree $j$ on $R^{N}$, which converges to $H$ on $B(\rho)$ (see, e.g., Brelot [3, Appendix]). In particular, the function $t \mapsto H(0, \ldots, 0, t)$ is given on the interval $(-\rho, \rho)$ by its Taylor series about 0 . Also, since by $(8)$ this function is odd, the Taylor series contains only odd powers of $t$. Thus we have a representation of the form

$$
H(0, \ldots, 0, t)=\sum_{j=0}^{\infty} a_{2 j+1} t^{2 j+1} \quad(-\rho<t<\rho) .
$$

We next aim to prove inductively that

$$
a_{2 j+1}=(-1)^{j+1}\left(\begin{array}{c}
j+\frac{N}{2}-1 \\
j
\end{array}\right) \int_{\tau(\rho)}\|y\|^{-N-2 j} d \mu(y)
$$

for each non-negative integer $j$. An argument by contradiction will then show that $\mu \equiv 0$ on $\tau(\rho)$, and the conclusions of Theorem 1 will then follow easily.

Throughout this paragraph $x$ denotes a point of $\alpha(\rho)$ with coordinates $(0, \ldots, 0, t)$. By (9) and (11),

$$
\begin{aligned}
t^{-1} h(x) & =\int_{\tau(\rho)}\left(t^{2}+\|y\|^{2}\right)^{-N / 2} d \mu(y)+a_{1}+O\left(t^{2}\right) \\
& \rightarrow \int_{\tau(\rho)}\|y\|^{-N} d \mu(y)+a_{1} \quad(t \rightarrow 0+),
\end{aligned}
$$

by monotone convergence. Hypothesis (3) with $m=1$ now implies that (12) holds with $j=0$. To proceed with the inductive proof of (12), we introduce the function $\phi$, defined by

$$
\phi(\theta)=(1+\theta)^{-N / 2} \quad(\theta>-1),
$$

and note that

$$
\frac{\phi^{(j)}(0)}{j !}=(-1)^{j}\left(\begin{array}{c}
j+\frac{N}{2}-1 \\
j
\end{array}\right) \quad(j=0,1,2, \ldots)
$$

and that by Taylor's theorem,

$$
\phi(\theta)-\sum_{j=0}^{k} \frac{\phi^{(j)}(0)}{j !} \theta^{j}=\frac{\theta^{k+1}}{k !} \int_{0}^{1}(1-\zeta)^{k} \phi^{(k+1)}(\theta \zeta) d \zeta \quad(\theta>-1, k=0,1,2, \ldots) .
$$


It is easy to see that $(-1)^{k+1} \phi^{(k+1)}$ is positive and decreasing on $(-1,+\infty)$. Hence it follows from (14) that if $\Phi_{k}$ is defined by

$$
\Phi_{k}(\theta)=\frac{(-1)^{k+1}}{\theta^{k+1}}\left(\phi(\theta)-\sum_{j=0}^{k} \frac{\phi^{(j)}(0)}{j !} \theta^{j}\right),
$$

then $\Phi_{k}$ is positive and decreasing on $(0,+\infty)$ and

$$
\lim _{\theta \rightarrow 0+} \Phi_{k}(\theta)=(-1)^{k+1} \frac{\phi^{(k+1)}(0)}{(k+1) !}
$$

Suppose now that (12) holds for $j=0, \ldots, k$. Then by (9) and (11),

$$
\begin{aligned}
h(x)= & t \int_{\tau(\rho)}\left(t^{2}+\|y\|^{2}\right)^{-N / 2} d \mu(y) \\
& -\sum_{j=0}^{k}(-1)^{j}\left(\begin{array}{c}
j+\frac{N}{2}-1 \\
j
\end{array}\right) t^{2 j+1} \int_{\tau(\rho)}\|y\|^{-N-2 j} d \mu(y) \\
& +\sum_{j=k+1}^{\infty} a_{2 j+1} t^{2 j+1} .
\end{aligned}
$$

Using (13), we can write this equation in the form

$$
\begin{aligned}
& \frac{h(x)}{t^{2 k+3}}=\frac{1}{t^{2 k+2}}\left\{\int_{\tau(\rho)}\|y\|^{-N}\left(\phi\left(\frac{t^{2}}{\|y\|^{2}}\right)-\sum_{j=0}^{k} \frac{\phi^{(j)}(0)}{j !}\left(\frac{t^{2}}{\|y\|^{2}}\right)^{j}\right) d \mu(y)\right\} \\
&+a_{2 k+3}+O\left(t^{2}\right) \\
&=(-1)^{k+1} \int_{\tau(\rho)}\|y\|^{-N-2 k-2} \Phi_{k}\left(\frac{t^{2}}{\|y\|^{2}}\right) d \mu(y)+a_{2 k+3}+O\left(t^{2}\right) .
\end{aligned}
$$

Since $\Phi_{k}$ is positive and decreasing on $(0,+\infty)$, it now follows from (15) that

$$
\lim _{t \rightarrow 0+} t^{-2 k-3} h(x)=\frac{\phi^{(k+1)}(0)}{(k+1) !} \int_{\tau(\rho)}\|y\|^{-N-2 k-2} d \mu(y)+a_{2 k+3} .
$$

This, together with (13) and hypothesis (3), implies that (12) holds for $j=k+1$, and the inductive proof of (12) is complete.

Now suppose that $\mu \not \equiv 0$ on $\tau(\rho)$. Choose $\sigma \in(0, \rho)$ such that $\mu(\tau(\sigma))>0$. Defining

$$
\lambda=\int_{\tau(\sigma)}\|y\|^{-N} d \mu(y)
$$

we have $\lambda>0$ and

$$
\int_{\tau(\sigma)}\|y\|^{-N-2 j} d \mu(y) \geq \lambda \sigma^{-2 j} \quad(j=0,1, \ldots) .
$$

Hence, by (12),

$$
\left|a_{2 j+1}\right| \geq \lambda\left(\begin{array}{c}
j+\frac{N}{2}-1 \\
j
\end{array}\right) \sigma^{-2 j} \geq \lambda \sigma^{-2 j},
$$

so that $\sum a_{2 j+1} t^{2 j+1}$ diverges when $t \geq \sigma$. As this series converges to $H(0, \ldots, 0, t)$ when $-\rho<t<\rho$, we have arrived at a contradiction. This shows that $\mu \equiv 0$ on $\tau(\rho)$. 
The representation (9) now reduces to $h=H$ on $\beta(\rho)$, and since $H=0$ on $\tau(\rho)$, (4) holds for each $y \in \tau(\rho)$. Also, (12) now gives $a_{2 j+1}=0$ for each $j$, so that by (11), $h=H=0$ on $\alpha(\rho)$. Since $\rho$ is an arbitrary number in $(0, r)$, this completes the proof.

\section{Proofs of the Corollaries}

Note first that $(0, \ldots, 0,1)$ lies in the spherical cap $C(d)$ for each $d \in(0,1)$. Hence if the hypotheses of Corollary 1 hold, then so do the hypotheses of Theorem 1 , and therefore $h$ has a harmonic continuation $\bar{h}$ to $B(r)$ such that $\bar{h}=0$ on $\tau(r)$. The function $\bar{h}$ is given on $B(r)$ by a series $\sum_{j=0}^{\infty} H_{j}$, where $H_{j}$ is a homogeneous harmonic polynomial of degree $j$ on $R^{N}$. We show by induction that $H_{j} \equiv 0$ for each $j$. First we have $H_{0} \equiv H_{0}(0)=\bar{h}(0)=0$. Now suppose that $H_{j} \equiv 0$ for each $j=0, \ldots, k$. If $z \in C\left(d_{k+1}\right)$ and $0<t<r$, then

$$
t^{-k-1} h(t z)=H_{k+1}(z)+O(t),
$$

and hypothesis $(6)$ implies that $H_{k+1}(z)=0$. Hence, by homogeneity, $H_{k+1}=0$ on the truncated cone $\left\{t z: z \in C\left(d_{k+1}\right), 0<t<r\right\}$, and therefore $H_{k+1} \equiv 0$. This completes the induction.

We note in passing that the hypotheses of Corollary 1 can be relaxed a little: instead of assuming (6) for each $z \in C\left(d_{m}\right)$, we need only suppose that (6) holds for each $z \in S_{m}$, where $S_{m}$ is a subset of the hemisphere $C(0)$ such that $(0, \ldots, 0,1) \in$ $S_{m}$ and the closure of $S_{m}$ has non-empty interior in the topology of $C(0)$.

To prove Corollary 2, note first that (7) implies (2), so that by Theorem 1 a function $h$ satisfying the hypotheses of Corollary 2 has a harmonic continuation $\bar{h}$ to $B(r)$ satisfying (5). The function $\partial \bar{h} / \partial x_{N}$ is also harmonic on $B(r)$ and if $y \in \tau(r)$, then

$$
\begin{aligned}
\frac{\partial \bar{h}}{\partial x_{N}}(y) & =\lim _{t \rightarrow 0+} h\left(y_{1}, \ldots, y_{N-1}, t\right) / t \\
& \geq \liminf _{x \rightarrow y, x \in \beta(r)} h(x) / x_{N} \geq 0 .
\end{aligned}
$$

It also follows from Theorem 1 that $h=0$ on $\alpha(r)$ and hence $\partial h / \partial x_{N}=0$ on $\alpha(r)$. We have now shown that the hypotheses of Theorem 1 are satisfied with $\partial h / \partial x_{N}$ in place of $h$. Hence $\partial h / \partial x_{N}$ has a harmonic continuation $H$ to $B(r)$ satisfying

$$
H\left(x_{1}, \ldots, x_{N-1},-x_{N}\right)=-\frac{\partial h}{\partial x_{N}}\left(x_{1}, \ldots, x_{N}\right) \quad(x \in \beta(r)) .
$$

The harmonic functions $H$ and $\partial \bar{h} / \partial x_{N}$ are both equal to $\partial h / \partial x_{N}$ on $\beta(r)$, and hence $H=\partial \bar{h} / \partial x_{N}$ on $B(r)$. Therefore, differentiating (5), we obtain

$$
H\left(x_{1}, \ldots, x_{N-1},-x_{N}\right)=\frac{\partial h}{\partial x_{N}}\left(x_{1}, \ldots, x_{N}\right) \quad(x \in \beta(r)) .
$$

Equations (16) and (17) imply that $H \equiv 0$, so that $\bar{h}(x)$ is independent of $x_{N}$. Since $\bar{h}=0$ on $\tau(r)$, it follows that $h(=\bar{h})=0$ on $\beta(r)$.

\section{OTHER DOMAINS}

We briefly indicate how results analogous to Theorem 1 and its corollaries can be proved with a spherical cap in place of the $(N-1)$-dimensional ball $\tau(r)$. In this section, the open ball of centre $x$ and radius $r$ in $R^{N}$ is denoted by $B(x, r)$, and 
its boundary is denoted by $S(x, r)$. Let $\rho, s$ be numbers such that $0<s<\rho$, let $x_{0}=(0, \ldots, 0,-\rho)$, and let $\Omega=B\left(x_{0}, \rho\right) \cap B(0, s)$. Suppose that $h$ is harmonic on $\Omega$ and let $h^{*}$ be the image of $h$ under the Kelvin transform relative to $S\left(2 x_{0}, 2 \rho\right)$, that is,

$$
h^{*}(x)=\left(\frac{2 \rho}{\left\|x-2 x_{0}\right\|}\right)^{N-2} h\left(x^{*}\right),
$$

where

$$
x^{*}=\frac{4 \rho^{2}\left(x-2 x_{0}\right)}{\left\|x-2 x_{0}\right\|^{2}}+2 x_{0} .
$$

Then $h^{*}$ is harmonic on the domain $\Omega^{*}=\left\{x^{*}: x \in \Omega\right\}$ (see, e.g., [4, p. 36]). It is easy to check that $\beta(r) \subseteq \Omega^{*} \subseteq \beta\left(r^{\prime}\right)$ for some $r, r^{\prime}>0$. Suppose now that

$$
\liminf _{x \rightarrow y, x \in \Omega} h(x) \geq 0 \quad\left(y \in S\left(x_{0}, \rho\right) \cap B(0, s)\right)
$$

and

$$
\liminf _{t \rightarrow 0-}|t|^{-m}|h(0, \ldots, 0, t)|=0 \quad(m=1,2, \ldots) .
$$

Then $h^{*}$ satisfies the hypotheses of Theorem 1 and hence $h^{*}=0$ on $\alpha(r)$ and $h^{*}$ has limit 0 at each point of $\tau(r)$. These conclusions imply that $h=0$ on the line segment $\{(0, \ldots, 0, t):-s<t<0\}$ and that $h$ has limit 0 at each point of the spherical cap $\left\{x^{*}: x \in \tau(r)\right\} \subset S\left(x_{0}, \rho\right)$.

Finally we give an example to show that Theorems BR and 1 may fail in a smooth domain.

Example. Let $\Omega=\left\{x: x_{N}>x_{1}^{3},\|x\|<1\right\}$. There exists a continuous, real-valued function $h$ on $\bar{\Omega}$, harmonic on $\Omega$, such that

(i) $h \geq 0$ on $\{x \in \partial \Omega:\|x\|<1\}$,

(ii) $\lim _{t \rightarrow 0+} t^{-m} h(0, \ldots, 0, t)=0 \quad(m=1,2, \ldots)$, but

(iii) $h>0$ on $\alpha(1) \cup\left\{x \in \partial \Omega:\|x\|<1, x_{N} \neq 0\right\}$,

(iv) $h$ has no harmonic continuation to any neighbourhood of 0 .

It is enough to work in the plane, for if we produce an example $h$ with $N=2$, then the function $\left(x_{1}, \ldots, x_{N}\right) \mapsto h\left(x_{1}, x_{N}\right)$ will be a corresponding example in $R^{N}$.

We identify $R^{2}$ with the complex plane $\mathbb{C}$ in the usual way and first define a function $h_{1}$ on the cut plane $\mathbb{C} \backslash\left\{i x_{2}: x_{2} \leq 0\right\}$ by

$$
h_{1}\left(r e^{i \theta}\right)=\operatorname{Re}\left(\exp \left(r^{-2 / 3} e^{-2(\theta+\pi) i / 3}\right)\right) \quad\left(r>0,-\frac{\pi}{2}<\theta<\frac{3 \pi}{2}\right) .
$$

Then $h_{1}$, being the real part of a regular function, is harmonic on its domain of definition. Also,

$$
\begin{aligned}
\left|h_{1}\left(r e^{i \theta}\right)\right| & \leq \exp \left(r^{-2 / 3} \cos \frac{2(\theta+\pi)}{3}\right) \\
& <\exp \left(-a r^{-2 / 3}\right) \quad\left(r>0,-\frac{\pi}{6}<\theta<\frac{7 \pi}{6}\right),
\end{aligned}
$$

where $a=-\cos \frac{5 \pi}{9}>0$. This implies that

(a) if we define $h_{1}(0)=0$, then the restriction of $h_{1}$ to $\bar{\Omega}$ is continuous on $\bar{\Omega}$,

(b) there exists a positive constant $A$ such that

$$
\left|h_{1}(z)\right| \leq A|z|^{4} \quad(z \in \bar{\Omega})
$$


(c) $\lim _{t \rightarrow 0+} t^{-m} h_{1}(i t)=0 \quad(m=1,2, \ldots)$.

Also,

(d) $h_{1}(i t)=\exp \left(-t^{-2 / 3}\right)>0 \quad(t>0)$.

Now define $h_{2}$ on $\mathbb{C}$ by $h_{2}\left(x_{1}+i x_{2}\right)=x_{1} x_{2}$. Then $h_{2}$ is harmonic on $\mathbb{C}$ and if $z=x_{1}+i x_{2} \in\{z \in \partial \Omega:|z|<1\}$, then

$$
h_{2}(z)=x_{1}^{4} \geq \frac{1}{4}\left(x_{1}^{2}+x_{1}^{6}\right)^{2}=\frac{1}{4}\left(x_{1}^{2}+x_{2}^{2}\right)^{2}=\frac{1}{4}|z|^{4} .
$$

Finally define $h=h_{1}+5 A h_{2}$ on $\bar{\Omega}$. Then $h$ is continuous on $\bar{\Omega}$ and harmonic on $\Omega$, and using the above properties of $h_{1}$ and $h_{2}$ we find that $h \geq 0$ on $\{z \in \partial \Omega:|z|<$ $1\} \cup\{i t: 0<t<1\}$ with equality only at 0 and

$$
\lim _{t \rightarrow 0+} t^{-m} h(i t)=0 \quad(m=1,2, \ldots) .
$$

Also it is clear that $h_{1}$ has no harmonic continuation to any neighbourhood of 0 , and therefore $h$ has no such continuation.

\section{REFERENCES}

[1] M. S. Baouendi, L. P. Rothschild, A local Hopf lemma and unique continuation for harmonic functions, Duke J. Math., Inter. Research Notices, 71 (1993), 245-251. MR 94i:31008

[2] M. S. Baouendi, L. P. Rothschild, Harmonic functions satisfying weighted sign conditions on the boundary, Ann. Inst. Fourier, Grenoble, 43 (1993), 1311-1318. MR 95c:35067

[3] M. Brelot, Éléments de la théorie classique du potentiel, Centre de documentation universitaire, Paris, 1965. MR 31:2412

[4] L. L. Helms, Introduction to potential theory, Wiley, New York, 1969. MR 41:5638

[5] A. Huber, On functions subharmonic in a half-space, Trans. Amer. Math. Soc., 82 (1956), 147-159. MR 17:1197b

[6] Ü. Kuran, Study of superharmonic functions in $R^{n} \times(0,+\infty)$ by a passage to $R^{n+3}$, Proc. London Math. Soc. (3), 20 (1970), 276-392. MR 41:5643

Department of Pure Mathematics, The Queen's University of Belfast, Belfast Bt7 1 NN, NorThern IRELAND

E-mail address: d.armitage@qub.ac.uk 